\title{
Reduction of Wastes and Costs Through the Modified Kanban System in Unit Storerooms of HGH: A Value-for-Money Quality Improvement Initiative
}

KENNETH JUN LOGRONO ( $\sim$ KLogrono@hamad.qa )

HAMAD MEDICAL CORPORATION https://orcid.org/0000-0002-7617-9794

BELAL SALEM ZU'BI

HAMAD MEDICAL CORPORATION

\section{Method Article}

Keywords: cost-efficient, Kanban, lean, logistics, quality improvement, supply chain, value improvement

Posted Date: November 17th, 2021

DOI: https://doi.org/10.21203/rs.3.rs-1070569/v1

License: (c) (1) This work is licensed under a Creative Commons Attribution 4.0 International License.

Read Full License 


\section{Abstract}

Most public health sectors in many countries were heavily burdened both financially and operationally as a result of the pandemic's new changes. Organizations have been pushed to develop innovative solutions to balance expenses and revenues through value-for-money initiatives as a result of erratic economic conditions.

Purchasing and supply distribution to the point of care account for approximately $30-40 \%$ of the hospital's operating budget. The demand-based ordering system, also known as the par system, has inherently resulted in increased waste due to obvious estimations for the quantity of items. Increased waste and costs have become a widespread issue in the healthcare supply chain, which was identified as a priority for improvement.

The project is aimed to reduce the costs by $50 \%$ by implementing the Kanban system in the unit storeroom of medical units within three months. The IHI Model of Improvement was undertaken to go through the implementation of the project. Several quality improvement tools were used to test different ideas of change and measurement of improvements were also made.

Following the adoption of the modified Kanban System, the costs of consumable item supply decreased by $69 \%$ from 16,000 QAR to 5,000 QAR. The percentage of wastes has reduced significantly to $0 \%$ while limiting the percentage of out stocking to a median of $1 \%$.

Kanban as a lean method, reduces wastes by controlling the flow of consumables within a supply chain. Eliminating or reducing waste is a cornerstone of lean management. The project's new evidence demonstrated significant and sustainable cost saving strategy by reducing waste and pre-quantifying items based on their usage and flow. The Kanban system ultimately improved nurses' productivity, streamlined the unit's stock supply, promoted value improvement, and helped increased end-user satisfaction.

\section{Problem}

The logistical workflow process from the central storeroom to the unit storeroom at Hamad General Hospital $(\mathrm{HGH})$ is a demand-based ordering system. The par system, also known as the eyeball system, is used by nurses to evaluate requests based on their par level. With the par system, estimation is apparent, and accuracy for the quantity of items is compromised as most nurses quantify the request by eyesight. 
Unnecessary inventory counts are also conducted by nursing staff based on consumption estimates, which frequently results in supply discrepancies that have a significant impact on patient care.

Waste is defined as consumables that were not used before their expiration date. Pre-implementation observations revealed that wastes are increasing among slow-moving items (i.e., consumables that are not typically used within a week or month) while becoming less common among fast-moving items (i.e., items which usage rate is rapid usually consumed within a week or two). Waste, however, can take various forms like increased time, effort, and unnecessary logistical activities such as idle inventory and item counting.

With Increasing wastes comes along with increasing costs, which have become a pervasive problem in the supply chain being a target area for improvement. The project aimed to reduce the costs by $50 \%$ by implementing the Kanban system in the unit storeroom of medical units after three months.

\section{Background}

Inventory management, purchasing, and supply distribution to the point of care are just a few of the activities that support patient care. These activities, referred to by many as healthcare logistics, or supply chain management, with aim to ensure the delivery of the right products where and when needed, at the quality and quantity required to provide services while preventing any stock disruptions (1). As these logistic services use approximately $30-40 \%$ of the hospital's operating budget (2), these activities are often cited as areas for improvement.

There have been several suggestions and projects to overcome the increasing wastes in the unit storerooms like the application of $5 \mathrm{~S}$ methodology where found not completely effective. There is no arguing that the $5 \mathrm{~S}$ process is one of the most valuable assets to lean but the $5 \mathrm{~S}$ is not a standalone program instead an ongoing process. Kanban is one of the most popular and widely used Lean tools (23). The system was adopted and modified so that it could be used in the HGH workflow. Kanban, which means "signboard" in Japanese, is a system for managing the overall supply chain by strategically and operationally connecting production demands and supply management.

This manufacturing-industry concept will be tested and applied in $\mathrm{HGH}$, nonetheless, the Kanban system as a lean tool, provided a straightforward intervention in overcoming the increased wastes and costs. It includes a streamlined process that eliminates non-value steps in the supply chain from central stores to 
nursing units (4), as well as a reorganization of the storerooms for easy tracking of consumables, thereby improving the value for money.

\section{Measurement}

The primary outcome measure was the weekly cost of supplied items (in QAR currency), which was aimed to be reduced by $50 \%$. This is calculated by adding the prices of kanbanized items at each supply time during each week period. Pre-implementation data collection focused on the costs per supply of consumables items from the main storeroom over a six-month period. According to the data, the average baseline cost was 16,000 QAR.

As part of the project's process measures, the percentage of wastage for both fast-moving and slowmoving items was calculated. Wastage is defined as an item that has reached its expiration date without being used and is likely to be thrown away or condemned. Slow-moving items like gastrointestinal tubes, wound dressing paraphernalia, operating hand scrubs, restraints, and IV fluids like dextrose-containing fluids are common causes of wastage. The number of items thrown away in the pre-Kanban system was alarming, according to the data. The goal is to reduce fast-moving item waste by ten percent and slowmoving item waste by twenty percent.

Furthermore, staff compliance is also a process measure. Compliance monitoring has been implemented to ensure that the Kanban guidelines and rules are followed. Due to the novelty of the concept and the effort required to transition from the old par system, an information drive, awareness sessions, and educational huddles about Kanban were significant to achieving a full compliance rate. The staff compliance rate is the percentage of entities or instances that conform to set Kanban rules and regulations and required to take at least five to ten random observations in a given week.

The Kanban system's out stocking monitoring is a critical balance measure. The number of items out of stock divided by the overall total number of items multiplied by a hundred, yields the out-stocking percentage. The goal is to reduce out stocking percentage by $50 \%$, starting with a baseline of $10 \%$.

\section{Design}

The implementation of the Kanban system started after forming a group of champions. This includes the staff nurses of one of the acute medical units along with the important stakeholders including a nurse manager, directors of nursing, executive leadership, and quality improvement specialist (see Figure 1). The champions are primarily responsible in ensuring timely implementation and adherence to the 
improvement methodology. A guideline was developed with the involvement of the key stakeholders, stipulating the specific steps in the Kanban process from requisition in the procurement software to the supply of consumables from the main store. There were four phases to the project's implementation.

\section{Classification of Items}

The first phase began with a classification of consumables based on their use in specific medical units. Consumables were divided into two categories- fast-moving and slow-moving items. Fast-moving items were those that were used and utilized within a week and frequently caused out-of-stocking, whereas slow-moving items were those that were rarely used. These items are frequently underutilized and may be close to expiration dates, posing a risk of becoming a waste. It is crucial to assess at the flow of consumables based on the occupancy rate of the unit and its scope of service. This will also aid the team in calculating quantities based on the utilization rate of each consumable.

\section{Kanbanize}

The next phase was a crucial step in where all items were Kanbanized. There were three prerequisites for kanbanizing an item: 1.) determine the item's utilization rate, 2.) determine the frequency of supply from the main store, and 3.) assess the sizes of the bin where items will be placed. In each week, the utilization rate is calculated based on the usage rate either by piece, by box, or per stated quantity. The supply of materials and items from the main store was usually once or twice per week in the Kanban process. This helped to derive and standardize the Kanban level calculation. The sizes of the bins are also important as they will provide us with an accurate estimate of how many items can be placed.

Moreover, the Kanban system had been modified into two sub-systems-- the divider method, and the bin system. The divider method employs a divider that serves as a signal, separating the Kanban level from the total bin quantity (number of items that a bin can accommodate). The Kanban quantity is usually calculated after determining the utilization rate. As a result, the Kanban quantity is always at least $20 \%$ of the total number of items that can fit into the bins. Conversely, the Kanban quantity will always be the same to total bin quantity in very fast-moving items (like blood extraction, butterflies, syringes, blood taps, saline solutions, and the like). This time, a bin system can be used to accommodate the high-volume flow of the items. It thus prevents out stocking incidences by employing a two- or three-bin system.

After the bin quantity and Kanban level have been determined, Kanban and bin labels are generated. The Kanban cards contained information such as the requisition order, the bin's predetermined quantity, the Kanban quantity level, and the item's name. These cards will serve as a signal for when the store's supply cue should be triggered. The Kanban board is also a useful tool because it serves as a means of communication among the nurses. The card will be removed and placed in the first section of the board once the bin quantity has reached its Kanban level (To Order Section). The cards will then be moved to 
the "Ordered" section once the nurse managers have completed the requisition, and if supplies arrived, they will be placed to the "Supplied" section of the board.

\section{Adopting 5S Method}

The third phase entailed implementing the $5 S$ methodology to efficiently organize and sort items. Sort, set in order, shine, and standardize by creating visual cues such as signs, labels, posters, floor marking tape, and Kanban boards; and sustain through training and education for nurses to ensure the project's longterm viability and effectiveness. It emphasized putting things where they belong and keeping the storeroom ergonomically efficient, making it easier for nurses to locate the consumables without wasting time.

\section{Education and Training}

Education of staff nurses and other end users (like housekeeping, clerks, store man, and nursing aides) about the Kanban process was done simultaneously along with the phases of implementation. The project champions met every day to discuss the Kanban guidelines, and weekly meetings have been conducted to discuss the improvement measures. Stakeholders like the executives and directors oversee the project's implementation on a regular basis by checking on staff knowledge of the new Kanban process.

\section{Strategy}

After three months, the SMART goal was to reduce costs by half by implementing the Kanban system in the storerooms of medical units. Six PDSA test cycles have been undertaken out as part of the project.

PDSA cycle 1: The initial intervention was to introduce the change concept of Kanban. This was accomplished by testing the concept on a small scale, beginning with five consumable items in the preparation room. This resulted in an increase in compliance rate from $50 \%$ to $80 \%$. Staff feedback emphasized the importance of developing novel methods of engaging staff through educational videos and activities.

PDSA Cycle 2: To increase compliance, robust Kanban education was implemented. The use of video lectures for Kanban, on-the-spot Q\&A from the leadership, and one-on-one walk-through methods kept the compliance rate at 80 percent. These activities will be maintained while they have proven to be effective and engaging. 
PDSA Cycle 3: After several weeks, the data suggests that compliance with Kanban rules was still between $80 \%$ and $85 \%$. The root cause of this problem was observed to be that Kanban and bin labels kept falling and that some of the information on the labels was misleading, causing staff confusion. This was immediately remedied by selecting sturdy tapes and materials to prevent falling. To make it more sustainable, a workshop order was devised that included Engineering in the placement of the labels on the bins.

PDSA Cycle 4: The goal of this PDSA is to improve staff compliance by correcting misleading information on bin labels and Kanban cards. Project champions double-checked and reviewed the information for each consumable in the requisition software (especially the requisition identification of each item). Despite being successful in increasing compliance to 90-100 percent, there were instances of out socking. As a result, the team revisited the Kanban and bin quantities, as well as the logistical process from such days of requisitions.

PDSA Cycle 5: The team re-quantified the items based on their utilization rate because the episode of out socking had been increasing. The out stocking is due to assigning a replenishment day (every Wednesday) where store delivery does not follow the exact timing, according to the root cause analysis. The changing scope of service is also an identified issue, as item classifications have changed, affecting the number of supplied consumables against their use, as well as flow issues in the department, as some units were transferred or closed due to the system-wide response to the COVID pandemic, so units must support each other by providing consumables every now and then until a specific unit becomes stable.

Eliminating the assigning day for replenishment or requisition had been an effective strategy for reducing episodes of out socking, particularly on IV fluids. The out-stocking percentage was clearly reduced to 0-1 percent after re-kanbanizing the IV fluids (moving from the divider method to the bin-system). A safety net in the Kanban process is the provision of "extra space"-a space where excess items from the supply will be placed so that nurses have something to take while waiting for the next supply to arrive.

PDSA Cycle 6: Due to the inevitable changing scope of service and increasing bed capacity of the units, there has been a fluctuating reduction in costs (ranging from 40-50 percent reduction). The Kanban level was re-quantified by computing based on the maximum bed capacity. After several weeks of implementing this PDSA, there was a noticeable stable reduction in costs; costs had been consistently reduced by $50-60 \%$ even up to the present. 


\section{Results}

Each subsequent intervention that was implemented had a significant effect on cost and waste reduction of consumable items. Data were collected for three months for each test cycle and the improvement measures were plotted on a run chart. This data was measured continuously over a period of one year from 2020.

The pre-implementation cost of consumable items from the previous par system is 16,000 QAR. After three months of pilot implementation, the weekly average cost has reduced by significantly 69 percent from the baseline data (see Graph 1). This significant reduction of costs has validated the fact that Kanban system as a lean tool improved the logistical process of the consumable item flow resulting to an average weekly cost of 4000 to 5000 QAR. classifying consumables into fast and slow-moving items, hence eliminating the unnecessary quantity of items, and implementing strategies to utilize items before it reaches the expiration date. Near-expiry items and expired items were very easy to identify. Prequantifying the items based on the unit's occupancy rate reduced the costs and made it easier to track the expiration date of the items. The hospital saved money by not having to discard expired items, and staff nurses had more time to do what they were hired to do-- to care for people.

There was also a special cause variation on week 6 where there was a sudden change of scope of service and logistical issues within the facility by increasing the bed capacity causing adding of items to accommodate other unit transfers. However, this was overcome through generating PDSAs and costs sustainably controlled within the target.

Graph 1 Costs per Supply in a weekly basis

Along with the cost reduction, data suggested an immediate and drastic reduction of wastage percentages of items and has been sustainable up until today. The goal is to reduce the fast- moving and slow-moving item wastage by $10 \%$. Significantly, wastage percentage for fast-moving items after three months of Kanban implementation is $0 \%$ from $30 \%$ and for slow-moving items has become consistently $0 \%$ from $20 \%$ (see Graphs 2 and 3). With a median wastage percentage of $0 \%$, the data showed sustainable improvement which reasons include 1.) optimizing the first expiry- first out system (FEFO), 2.) adjusting the item quantity of slow-moving items to a limit (usually $50 \%$ cut from the previous bin quantity), and 3.) studying the flow or movement of the consumables (slow and fast-moving items).

\section{Graph 2 Percentage of Wastes in Fast-Moving Items}

\section{Graph 3 Percentage of Wastes in Slow-Moving Items}

Staff compliance with Kanban was initially a major issue until the team developed effective methods for orienting the staff nurses through video campaigns and education. The median compliance rate was found to be $80 \%$, indicating that there has been a sustainable shift (see Graph 4). On Week 5, when new joiners returning from COVID facilities were alienated from the system, there was a noticeable drop in compliance. The concern was alleviated by a robust information drive and online reminders, and the 
Kanban system is fully implemented not only by staff nurses but also by charge nurses, housekeepers, nursing aides, and clerks.

\section{Graph 4 Staff Compliance to Kanban Guidelines}

There was a decreasing trend of out stocking percentage from week 3 to week 9 (see Graph 5). From the baseline of out stocking percentage of $10 \%$, post- intervention data showed a median out stocking of only $1 \%$. This significant reduction was providing an area for excess items called the "extra space", and periodic re- calibration of Kanban quantity especially for fast- moving items like the IV fluids.

Graph 5 Percentage of Out Stocking of Consumable Items

\section{Lessons and Limitations}

The goal of the project was to reduce the cost per supply of consumables by reducing waste and prequantifying items based on their usage and flow. Rather than aiming for short-term interventions, the project aimed for sustainable results. The new Kanban process was intended to be implemented on a larger scale, across the entire facility. To accomplish this, the process is designed to work in a variety of settings, including emergency, critical care units, and outpatient department. Nevertheless, the importance of involving both stakeholders and end-users was a key takeaway from the implementation.

It is critically important to identify the right people and involve the top-level leadership to provide oversight and support. Staff nurses will realize that the project is significant if they see that their leaders are committed to it. Leaders promote psychological meaningfulness to their staff by emphasizing that value for money initiatives are highly making sense within the organization (5). Leadership buy-in based from the learnings of the project helped staff nurses to balance options both in providing support and trust to the initiative. Furthermore, involving end-users not only staff nurses but also the housekeeping, nursing aides, and clerks helped ensure that the change idea makes sense in terms of reducing costs and eliminating wastes. End-user buy-in, on the other hand is equally essential. As they are involved in the day-to-day process and have a lot of experience gain from the deficiencies and successes of the change concept, they can eventually work around through generating PDSAs.

The staff and end-users' buy-in had become a struggle to implement and sustain in the first few weeks. The team thought it was ambitious to overhaul the supply chain's old system and replace it with a newer and more innovative Kanban concept. This could be due to the fact that majority of the nurses had highly variable demographics, such as age (young to old staff), change psychology (laggards, adopters, and passives), and leadership influence. These factors had been overcome through staff engagement and top-leadership involvement. End-users most especially nurses were invited to participate in conceptualizing the idea from process to guidelines, from implementation to practice. They were also engaged in generating change concepts through PDSAs and often involved in daily huddles and periodic discussions with the leadership. 
Furthermore, adopting a lean concept from manufacturing industry like Kanban to the healthcare supply chain had been a challenge, as the team had to modify steps and improve the process to make it relevant and applicable to the local system. Given the goal of a system-wide change to supply chain, Kanban has been designed to be feasible and work in all areas across the facility. To ensure that the Kanban process was running smoothly, the pilot team paid periodic surprise visits to each unit that had adopted it. Improvement measures have been documented in a centralized file folder accessible to all project champions. Top management will be able to see the improvement stories over the course of the sustainability period in this way. Similar trends and shifts in cost and waste reduction have been observed in other units that have recently adopted the Kanban system, as have the pilot units.

In cultivating a continuous improvement culture, it is critical to understand the concept of buy-in before trying to convince others to participate. Staff and leadership buy-in to innovation and change is more important than ever in the success of any healthcare organization (5). Ultimately, the project demonstrated how buy-in and full engagement drive the aim to a success. The more buy-in from both ends demonstrate in support of the change, the more likely the change will be successful and sustained over time.

\section{Conclusion}

It was clearly identified that wastes have been increasing in the overlooked process of supply chain where most of the hospital's operating budget is sourced out. The application of lean methodology like Kanban, originally from the manufacturing industry conclusively can reduce costs and limit the inherent wastes found in the healthcare systems. Nursing staff had control over the wastes from previous system of handling the consumable items.

The project demonstrated critical and sustainable improvements in lowering costs by 69 percent. The adoption of the Kanban System and the application of 5 S clearly reduced various types of waste, not only in terms of cost, but also of time, effort, and unnecessary logistical activities. It significantly improved workflow logistics from store requisition process to supply delivery in the point of care, resulting in greater efficiency and a more streamlined process.

\section{Declarations}

Acknowledgement

We would like to acknowledge the contributions of the project champions across $\mathrm{HGH}$, alongside with the full support of Ms Khadija Al-Shukaili, Mr Aidan Kehoe, Dr Yousuf Maslamani and Mr Esmat Swallmeh.

Without their leadership and full commitment this project would not have been possible.

Declaration of interests Nothing to declare. 
Ethical approval Formal ethical approval was not indicated for this project.

\section{References}

1. Escobar, V.G., Bourque, S., \& Godino-Gallego, N. (2015). Hospital Kanban system implementation: Evaluating satisfaction of nursing personnel. Science Direct Journal. 21(3). Pp. 101-110. Retrieved from https://doi.org/10.1016/j.iedee.2014.12.001

2. Landry, S., Blouin, J.P., and Beaulieu, M. (2013). The challenges of hospital supply chain management from central stores to nursing units. B.T. Denton (Ed.), Handbook of healthcare operations management: Methods and applications, 184, Springer Science Business Media, New York, pp. 465-482

3. Adams, S.G., \& Ruiz-Ulloa, B.C. (2003). An investigation of personnel issues affecting Kanban performance: A case study. Engineering Management Journal, 15 (4). pp. 19-27

4. Chalice., R. (2007). Improving healthcare using Toyota lean production methods: $\mathbf{4 6}$ steps for improvement. American Society for Quality Press, Milwaukee, WI. Retrieved from https://doi.org/10.1016/j.iedee.2014.12.001

5. French-Bravo, M., Crow, G., (March 19, 2015) "Shared Governance: The Role of Buy-In In Bringing About Change" OJIN: The Online Journal of Issues in Nursing Vol. 20 No. 2. https://doi.org/10.3912/OJIN.Vol20No02PPT02

\section{Graphs}

Graphs 1 - 5 can be found in the supplementary files section.

\section{Figures}




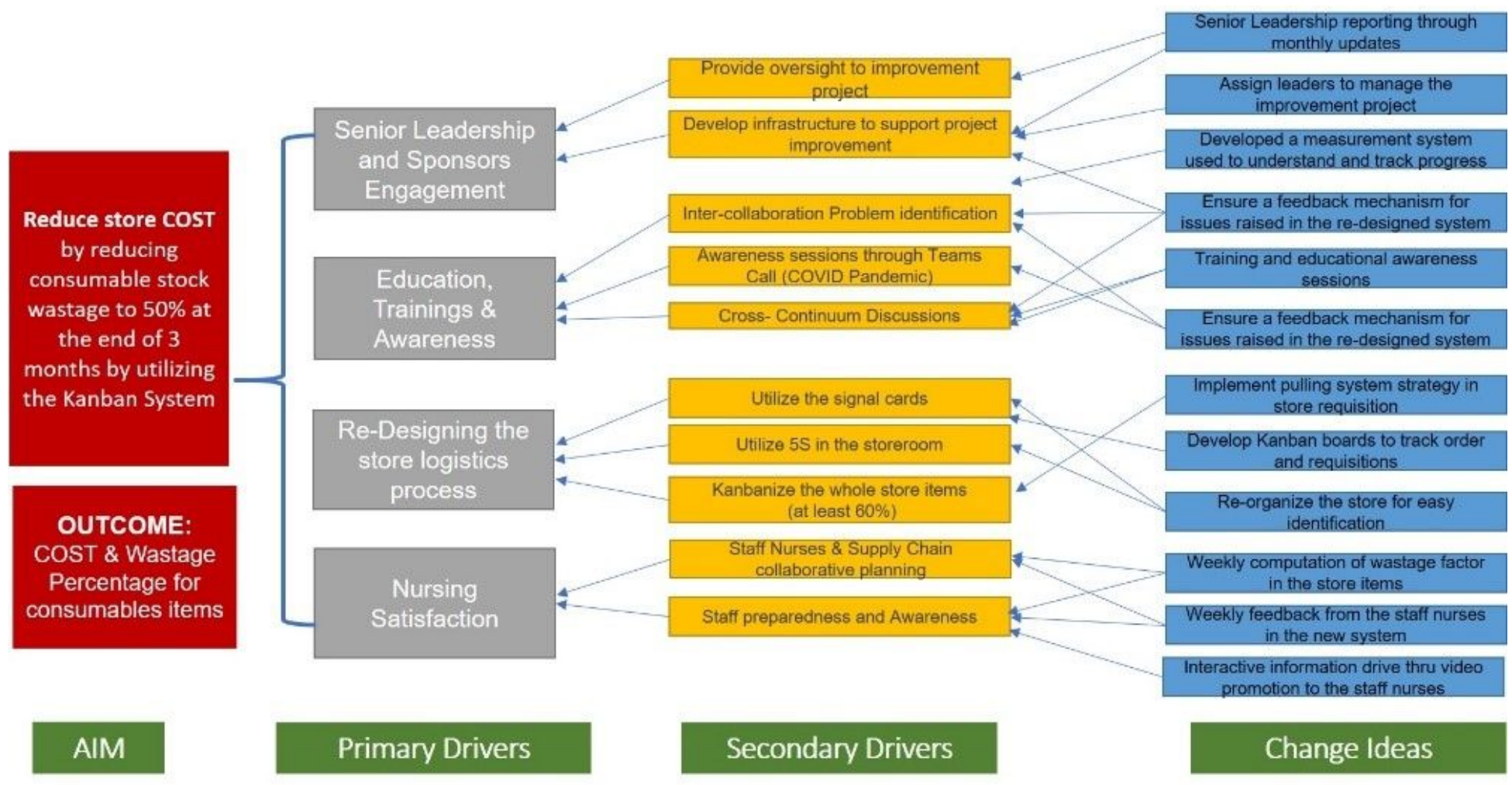

\section{Figure 1}

Figure 1 Driver Diagram

\section{Supplementary Files}

This is a list of supplementary files associated with this preprint. Click to download.

- g1.jpg

- PercentageofWastesFast.png

- StaffCompliance.jpg

- PercentageofWastesSlow.jpg

- OutStocking.jpg 